\title{
Analytical Blowup Solutions to the 2-dimensional Isothermal Euler-Poisson Equations of Gaseous Stars II
}

\author{
YUEN MANWAI* \\ Department of Applied Mathematics, \\ The Hong Kong Polytechnic University, \\ Hung Hom, Kowloon, Hong Kong
}

Revised 29-July-2009

\begin{abstract}
This article is the continued version of the analytical blowup solutions for 2-dimensional Euler-Poisson equations [12]. With extension of the blowup solutions with radial symmetry for the isothermal Euler-Poisson equations in $R^{2}$, other special blowup solutions in $R^{2}$ with non-radial symmetry are constructed by the separation method. We notice that the results are the first evolutionary solutions with non-radial symmetry for the system.

Key words: Analytical Solutions, Euler-Poisson Equations, Isothermal, Blowup, Non-radial Symmetry, Line Source or Sink
\end{abstract}

\section{Introduction}

The evolution of a self-gravitating fluid (gaseous stars) can be formulated by the isentropic EulerPoisson equations of the following form:

$$
\left\{\begin{aligned}
\rho_{t}+\nabla \cdot(\rho \vec{u}) & =0, \\
(\rho \vec{u})_{t}+\nabla \cdot(\rho \vec{u} \otimes \vec{u})+\nabla P & =-\rho \nabla \Phi, \\
\Delta \Phi(t, \vec{x}) & =\alpha(N) \rho,
\end{aligned}\right.
$$

where $\alpha(N)$ is a constant related to the unit ball in $R^{N}: \alpha(1)=2 ; \alpha(2)=2 \pi$ and For $N \geq 3$,

$$
\alpha(N)=N(N-2) V(N)=N(N-2) \frac{\pi^{N / 2}}{\Gamma(N / 2+1)},
$$

*E-mail address: nevetsyuen@hotmail.com 
where $V(N)$ is the volume of the unit ball in $R^{N}$ and $\Gamma$ is the Gamma function. And as usual, $\rho=\rho(t, \vec{x})$ and $\vec{u}=\vec{u}(t, \vec{x})=\left(u_{1}, u_{2}, \ldots, u_{N}\right) \in \mathbf{R}^{N}$ are the density and the velocity respectively.

In the above system, the self-gravitational potential field $\Phi=\Phi(t, \vec{x})$ is determined by the density $\rho$ through the Poisson equation.

The equation (11) 3 is the Poisson equation through which the gravitational potential is determined by the density distribution of the density itself. Thus, we call the system (11) the EulerPoisson equations. The equations can be viewed as a prefect gas model. The function $P=P(\rho)$ is the pressure. The $\gamma$-law can be applied on the pressure $P(\rho)$, i.e.

$$
P(\rho)=K \rho^{\gamma}:=\frac{\rho^{\gamma}}{\gamma}
$$

which is a commonly the hypothesis. The constant $\gamma=c_{P} / c_{v} \geq 1$, where $c_{P}, c_{v}$ are the specific heats per unit mass under constant pressure and constant volume respectively, is the ratio of the specific heats, that is, the adiabatic exponent in (3). In particular, the fluid is called isothermal if $\gamma=1$. It can be used for constructing models with non-degenerate isothermal cores, which have a role in connection with the so-called Schonberg-Chandrasekhar limit 8 .

The system can be rewritten as

$$
\left\{\begin{aligned}
\rho_{t}+\nabla \cdot \vec{u} \rho+\nabla \rho \cdot \vec{u} & =0 \\
\rho\left(\frac{\partial u_{i}}{\partial t}+\sum_{k=1}^{N} u_{k} \frac{\partial u_{i}}{\partial x_{k}}\right)+\frac{\partial}{\partial x_{i}} P(\rho) & =-\rho \frac{\partial}{\partial x_{i}} \Phi(\rho), \text { for } i=1,2, \ldots N \\
\Delta \Phi(t, x) & =\alpha(N) \rho .
\end{aligned}\right.
$$

For $N=3$, (4) is a classical (non-relativistic) description of a galaxy, in astrophysics. See [2], 3] and $[8]$ for a detail about the system.

For the local existence results about the system were shown in [9, [1] and [5]. In particular, the radially symmetric solutions can be expressed by

$$
\rho(t, \vec{x})=\rho(t, r) \text { and } \vec{u}(t, \vec{x})=\frac{\vec{x}}{r} V(t, r):=\frac{\vec{x}}{r} V
$$

where the radial diameter $r:=\left(\sum_{i=1}^{N} x_{i}^{2}\right)^{1 / 2}$. Historically in astrophysics, Goldreich and Weber constructed the analytical blowup (collapsing) solutions of the 3-dimensional Euler-Poisson equations for $\gamma=4 / 3$ for the non-rotating gas spheres [6]. After that, Makino [10] obtained the rigorously mathematical proof of the existence of such kind of blowup solutions. Besides, Deng, Xiang and Yang extended the above blowup solutions in $R^{N}(N \geq 3)$ [4]. Then, Yuen obtained the blowup solutions in $R^{2}$ with $\gamma=1$ by a new transformation [12. The family of the analytical solutions are rewritten as 
For $N \geq 3$ and $\gamma=(2 N-2) / N$, in [4]

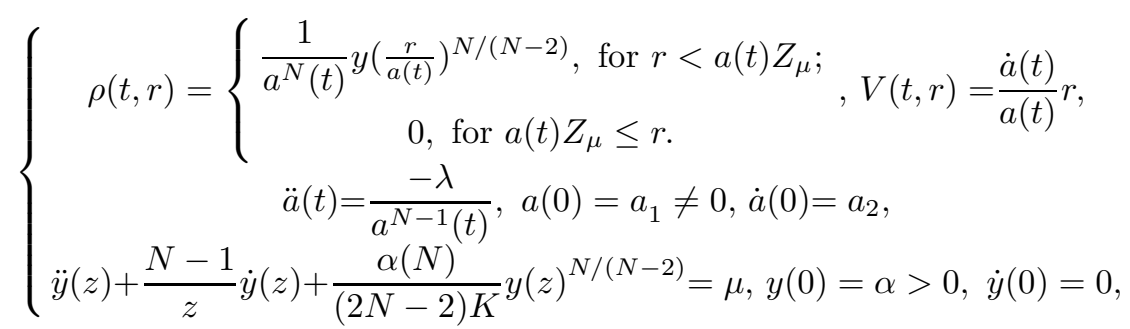

where $\mu=[N(N-2) \lambda] /(2 N-2) K$ and the finite $Z_{\mu}$ is the first zero of $y(z)$;

For $N=2$ and $\gamma=1$, in 12 ]

$$
\left\{\begin{array}{c}
\rho(t, r)=\frac{1}{a^{2}(t)} e^{y(r / a(t))}, V(t, r)=\frac{\dot{a}(t)}{a(t)} r \\
\ddot{a}(t)=\frac{-\lambda}{a(t)}, a(0)=a_{1}>0, \dot{a}(0)=a_{2} \\
\ddot{y}(z)+\frac{1}{z} \dot{y}(z)+\frac{\alpha(2)}{K} e^{y(z)}=\mu, y(0)=\alpha, \dot{y}(0)=0,
\end{array}\right.
$$

where $K>0, \mu=2 \lambda / K$ with a sufficiently small $\lambda$ and $\alpha$ are constants.

And for other special blowup solutions, the readers may see the details in [1].

Very recently, Yuen extended the above solutions to the pressureless Navier-Stokes-Poisson equations with density-dependent viscosity in [13]:

$$
\left\{\begin{aligned}
\rho_{t}+V \rho_{r}+\rho V_{r}+\frac{N-1}{r} \rho V & =0, \\
\rho\left(V_{t}+V V_{r}\right)+\frac{\alpha(N) \rho}{r^{N-1}} \int_{0}^{r} \rho(t, s) s^{N-1} d s & =\left[\kappa \rho^{\theta}\right]_{r}\left(\frac{N-1}{r} V+V_{r}\right)+\left(\kappa \rho^{\theta}\right)\left(V_{r r}+\frac{N-1}{r} V_{r}+\frac{N-1}{r^{2}} V\right) .
\end{aligned}\right.
$$

However, the known solutions are all in radial symmetry. In this paper, we are able to obtain the similar results to the non-radially symmetric cases for the 2-dimensional Euler-Poisson equations (4) in the following theorem:

Theorem 1 For the 2-dimensional isothermal Euler-Poisson equations (4), there exists a family of solutions,

$$
\left\{\begin{array}{c}
\rho(t, x, y)=\frac{1}{a(t)^{2}} e^{-\frac{\Phi\left(\frac{A x+B y}{a(t)}\right)}{K}+C}, \vec{u}(t, x, y)=\frac{\dot{a}(t)}{a(t)}(x, y), \\
a(t)=a_{1}+a_{2} t, \\
\ddot{\Phi}(s)-\epsilon^{*} e^{-\frac{\Phi(s)}{K}}=0, \Phi(0)=\alpha, \dot{\Phi}(0)=\beta,
\end{array}\right.
$$

where $A, B, a_{1} \neq 0, a_{2}, \frac{2 \pi e^{C}}{A^{2}+B^{2}}=\epsilon^{*}>0$ with that $A$ and $B$ are not both $0, \alpha$ and $\beta$ are constants. In particular, $a_{1}>0$ and $a_{2}<0$, the solutions (9) blow up in the finite time $T=-a_{2} / a_{1}$.

Remark 2 The solutions (9) is a line source or sink in terms of cylindrical coordinates, when we take $z$ direction to lie alone the characteristic line of the source or sink. For the physical significance of such kind of solutions, the interested readers may refer.P.409-410 of [7] for details. 


\section{Separable Blowup Solutions}

Before presenting the proof of Theorem 1 we prepare some lemmas first.

Lemma 3 For the continuity equation (4) $)_{1}$ in $R^{2}$, there exist solutions,

$$
\rho(t, x, y)=\frac{f\left(\frac{A x+B y}{a(t)}\right)}{a^{2}(t)}, \vec{u}(t, x, y)=\frac{\dot{a}(t)}{a(t)}(x, y),
$$

where the scalar function $f(s) \geq 0 \in C^{1}$ and $a(t) \neq 0 \in C^{1}$.

Proof. We plug the solutions (9) into the continuity equation (4) 1 ,

$$
\begin{aligned}
& \rho_{t}+\nabla \cdot \vec{u} \rho+\nabla \rho \cdot \vec{u} \\
& =\frac{\partial}{\partial t}\left[\frac{f\left(\frac{A x+B y}{a(t)}\right)}{a(t)^{2}}\right]+\nabla \cdot \frac{\dot{a}(t)}{a(t)}(x, y) \frac{f\left(\frac{A x+B y}{a(t)}\right)}{a(t)^{2}}+\nabla \frac{f\left(\frac{A x+B y}{a(t)}\right)}{a(t)^{2}} \cdot \frac{\dot{a}(t)}{a(t)}(x, y) \\
& =\frac{-2 \dot{a}(t)}{a(t)^{3}} f\left(\frac{A x+B y}{a(t)}\right)+\frac{1}{a(t)^{2}} \frac{\partial}{\partial t} f\left(\frac{A x+B y}{a(t)}\right)+\frac{\dot{a}(t)}{a(t)}\left(\frac{\partial}{\partial x} x+\frac{\partial}{\partial y} y\right) \frac{f\left(\frac{A x+B y}{a(t)}\right)}{a(t)^{2}} \\
& +\frac{\dot{a}(t)}{a(t)}\left[\frac{\partial}{\partial x} \frac{f\left(\frac{A x+B y}{a(t)}\right)}{a(t)^{2}} \cdot x+\frac{\partial}{\partial y} \frac{f\left(\frac{A x+B y}{a(t)}\right)}{a(t)^{2}} \cdot y\right] \\
& =\frac{-2 \dot{a}(t)}{a(t)^{3}} f\left(\frac{A x+B y}{a(t)}\right)-\frac{1}{a(t)^{2}} \dot{f}\left(\frac{A x+B y}{a(t)}\right) \frac{(A x+B y) \dot{a}(t)}{a(t)^{2}}+2 \frac{\dot{a}(t)}{a(t)} \frac{f\left(\frac{A x+B y}{a(t)}\right)}{a(t)^{2}} \\
& +\frac{\dot{a}(t)}{a(t)}\left[\frac{\dot{f}\left(\frac{A x+B y}{a(t)}\right)}{a(t)^{2}} \frac{A x}{a(t)}+\frac{\dot{f}\left(\frac{A x+B y}{a(t)}\right)}{a(t)^{2}} \frac{B y}{a(t)}\right] \\
& =0 \text {. }
\end{aligned}
$$

The proof is completed.

On the other hand, we may use the fixed point theorem to show the local existence of the following ordinary differential equation:

Lemma 4 There exists a sufficiently small $x_{0}>0$, such that the equation

$$
\left\{\begin{array}{l}
\ddot{\Phi}(s)-\epsilon^{*} e^{-\frac{\Phi(s)}{K}}=0 \\
\Phi(0)=\alpha, \dot{\Phi}(0)=\beta
\end{array}\right.
$$

where $\epsilon^{*}>0, \alpha$, and $\beta$ are constants, has a solution $\Phi=\Phi(s) \in C^{2}\left[0, s_{0}\right]$.

Proof. We integrate the equation (18) once:

$$
\dot{\Phi}(s)=-\int_{0}^{s} K \epsilon^{*} e^{-\frac{\Phi(\eta)}{K}} d \eta+\beta .
$$


And set

$$
f(s, \Phi(s))=-\int_{0}^{s} K \epsilon^{*} e^{-\frac{\Phi(\eta)}{K}} d \eta+\beta .
$$

then for any $s_{0}>0$, we get $f \in C^{1}\left[0, s_{0}\right]$. and for any $\Phi_{1}, \Phi_{2} \in C^{2}\left[0, s_{0}\right]$, we have,

$$
\left|f\left(s, \Phi_{1}(s)\right)-f\left(s, \Phi_{2}(s)\right)\right|=K \epsilon^{*}\left|\int_{0}^{s}\left(e^{-\frac{\Phi_{1}(\eta)}{K}}-e^{-\frac{\Phi_{2}(\eta)}{K}}\right) d \eta\right| .
$$

As $e^{y}$ is a $C^{1}$ function of $y$, we can show that the function $e^{y}$, is Lipschitz-continuous. And we get,

$$
\left|f\left(s, \Phi_{1}(s)\right)-f\left(s, \Phi_{2}(s)\right)\right|=O(1) \int_{0}^{s} \mid\left(\Phi_{1}(\eta)-\Phi_{2}(\eta)\left|d \eta \leq O(1) s_{0} \sup _{0 \leq s \leq s_{0}}\right| \Phi_{1}(s)-\Phi_{2}(s) \mid .\right.
$$

We let

$$
T \Phi(s)=\alpha+\int_{0}^{s} f(\eta, \Phi(\eta)) d \eta
$$

We have $T \Phi \in C\left[0, s_{0}\right]$ and

$$
\left|T \Phi_{1}(s)-T \Phi_{2}(s)\right|=\left|\int_{0}^{s} f\left(\eta, \Phi_{1}(\eta)\right) d \eta-\int_{0}^{s} f\left(\eta, \Phi_{2}(\eta)\right) d \eta\right| \leq O(1) s_{0} \sup _{0 \leq s \leq s_{0}}\left|\Phi(s)_{1}-\Phi(s)_{2}\right| .
$$

By choosing $s_{0}>0$ to be a sufficiently small number, such that $O(1) s_{0}<1$, this shows that the mapping $T: C\left[0, s_{0}\right] \rightarrow C\left[0, s_{0}\right]$, is a contraction with the sup-norm. By the fixed point theorem, there exists a unique $\Phi(s) \in C\left[0, s_{0}\right]$, such that $T \Phi(s)=\Phi(s)$. The proof is completed.

And we need another lemma to show the global existence of the ordinary differential equation (18).

Lemma 5 The equation,

$$
\left\{\begin{array}{l}
\ddot{\Phi}(s)-\epsilon^{*} e^{-\frac{\Phi(s)}{K}}=0, \\
\Phi(0)=\alpha, \dot{\Phi}(0)=\beta,
\end{array}\right.
$$

where $\epsilon^{*}>0, \alpha$ and $\beta$ are constants, has a solution in $(-\infty,+\infty)$ and $\lim _{s \rightarrow \pm \infty} \Phi(s)=+\infty$.

Proof. We prove the case $\dot{\Phi}(0)=0$ first. By integrating (25), we have,

$$
\dot{\Phi}(s)=\epsilon^{*} \int_{0}^{s} e^{-\frac{\Phi(\eta)}{K}} d \eta \geq 0 .
$$

Thus, for $0<s<s_{0}, \Phi(s)$ has a uniform lower bound

$$
\Phi(s) \geq \Phi(0)=\alpha .
$$

As we obtained he local existence in Lemma 4 there are two possibilities:

(1) $\Phi(s)$ only exists in some finite interval $\left[0, s_{0}\right]$ : (1a) $\lim _{s \rightarrow s_{0-}} \Phi(s)=+\infty$; (1b) $\Phi(s)$ has an uniformly upper bound, i.e. $\Phi(s) \leq \alpha_{0}$ for some constant $\alpha_{0}$.

$(2) \Phi(s)$ exists in $[0,+\infty)$ : (2a) $\lim _{s \rightarrow+\infty} \Phi(s)=+\infty$; $(2 \mathrm{~b}) \Phi(s)$ has an uniformly lower bound, i.e. $\Phi(s) \leq \alpha$ for some constant $\alpha_{0}$. 
We claim that possibility (1) does not exist. We need to reject (1b) first: If the statement (1b) is true, (26) becomes

$$
\epsilon^{*} s e^{-\frac{\alpha_{0}}{K}} \geq \epsilon^{*} \int_{0}^{s} e^{-\frac{\Phi(\eta)}{K}} d \eta=\dot{\Phi}(s)
$$

Thus, $\dot{\Phi}(s)$ is bounded in $\left[0, s_{0}\right]$. Therefore, we can use the fixed point theorem again to obtain a large domain of existence, such that $\left[0, s_{0}+\delta\right]$ for some positive number $\delta$. There is a contradiction. Therefore, (1b) is rejected.

Next, we do not accept (1a) because of the following reason: It is impossible that $\lim _{s \rightarrow s_{0-}} \Phi(s)=+\infty$, as from (28), $\dot{\Phi}(x)$ has a upper bound in $\left[0, s_{0}\right]$ :

$$
\epsilon^{*} s e^{-\frac{\alpha_{0}}{K}} \geq \dot{\Phi}(s)
$$

Thus, (29) becomes,

$$
\begin{aligned}
\Phi\left(s_{0}\right) & =\Phi(0)+\epsilon^{*} \int_{0}^{s_{0}} \dot{\Phi}(\eta) d \eta \\
& \leq \alpha+\epsilon^{*} \int_{0}^{s_{0}} s e^{-\frac{\alpha_{0}}{K}} d \eta \\
& =\alpha+\epsilon^{*} s_{0}^{2} e^{-\frac{\alpha_{0}}{K}} .
\end{aligned}
$$

Since $\Phi(s)$ is bounded upper in $\left[0, s_{0}\right]$, it contracts the statement (1a), such that $\lim _{s \rightarrow s_{0}-} \Phi(s)=+\infty$. So, we can exclude the possibility (1).

We claim that the possibility $(2 \mathrm{~b})$ does not exist. It is because

$$
\dot{\Phi}(s)=\epsilon^{*} \int_{0}^{s} e^{-\frac{\Phi(\eta)}{K}} d \eta \geq \epsilon^{*} \int_{0}^{s} e^{-\frac{\alpha_{0}}{K}} d \eta=\epsilon^{*} e^{-\frac{\alpha_{0}}{K}} s .
$$

Then, we have,

$$
\Phi(s) \geq \alpha+\epsilon^{*} e^{-\frac{\alpha_{0}}{K}} s .
$$

By letting $s \rightarrow+\infty$, (34) turns out to be,

$$
\Phi(s)=+\infty
$$

Since a contradiction is established, we exclude the possibility (2b). Thus, the equation (25) exists in $[0,+\infty)$ and $\lim _{s \rightarrow+\infty} \Phi(s)=+\infty$. Due to the solution is symmetric about $s=0$, we have $\lim _{s \rightarrow-\infty} \Phi(s)=+\infty$.

For the case $\dot{\Phi}(0)=\beta$, these exist constants $\tilde{s}$ and $\alpha_{\tilde{s}}$, such that $\Phi(0)=\beta$ in the ordinary differential equation:

$$
\left\{\begin{array}{l}
\ddot{\Phi}(s)-\epsilon^{*} e^{-\frac{\Phi(s)}{K}}=0, \\
\Phi(\tilde{s})=\alpha_{\tilde{s}}, \dot{\Phi}(\tilde{s})=0 .
\end{array}\right.
$$

The above fact is due to the transformation $z=s-\tilde{s}$ to have

$$
\left\{\begin{array}{l}
\ddot{\Phi}(z)-\epsilon^{*} e^{-\frac{\Phi(z)}{K}}=0, \\
\Phi(0)=\alpha_{\tilde{s}}, \dot{\Phi}(0)=0 .
\end{array}\right.
$$


Then we get $\lim _{z \rightarrow \pm \infty} \Phi(z)=\lim _{s \rightarrow \pm \infty} \Phi(s)=+\infty$ with the previous result and the continuity of the solutions (36) to show the lemma is true. This completes the proof.

On the other hand, the following lemma handles the Poisson equation (4) 3 for our solutions (9):

Lemma 6 The solutions,

$$
\rho=\frac{1}{a(t)^{2}} e^{-\frac{\Phi\left(\frac{A x+B y}{a(t)}\right)}{K}+C}
$$

with the second-order ordinary differential equation:

$$
\ddot{\Phi}(s)-\epsilon^{*} e^{-\frac{\Phi(s)}{K}}=0, \Phi(0)=\alpha, \dot{\Phi}(0)=\beta,
$$

where $s:=(A x+B y) / a(t)$ and $C, \frac{2 \pi e^{C}}{A^{2}+B^{2}}=\epsilon^{*}>0, \alpha$ and $\beta$ are constants, fit into the Poisson equation (4) $)_{3}$ in $R^{2}$.

Proof. We check that our potential function $\Phi(t, x, y)$ satisfies the Poisson equation (4) 3 :

$$
\begin{aligned}
& \Delta \Phi(t, x, y)-2 \pi \rho \\
& =\nabla \cdot \nabla \Phi\left(\frac{A x+B y}{a(t)}\right)-\frac{2 \pi}{a(t)^{2}} e^{-\frac{\Phi\left(\frac{A x+B y}{a(t)}\right)}{K}+C} \\
& =\nabla \cdot\left[\dot{\Phi}\left(\frac{A x+B y}{a(t)}\right) \frac{A}{a(t)}, \dot{\Phi}\left(\frac{A x+B y}{a(t)}\right) \frac{B}{a(t)}\right]-\frac{2 \pi}{a(t)^{2}} e^{-\frac{\Phi\left(\frac{A x+B y}{a(t)}\right)}{K}+C} \\
& =\frac{\partial}{\partial x}\left[\dot{\Phi}\left(\frac{A x+B y}{a(t)}\right) \frac{A}{a(t)}\right]+\frac{\partial}{\partial y}\left[\dot{\Phi}\left(\frac{A x+B y}{a(t)}\right) \frac{B}{a(t)}\right]-\frac{2 \pi}{a(t)^{2}} e^{-\frac{\Phi\left(\frac{A x+B y}{a(t)}\right)}{K}+C} \\
& =\frac{A^{2}+B^{2}}{a(t)^{2}}\left(\ddot{\Phi}(s)-\frac{2 \pi e^{C}}{A^{2}+B^{2}} e^{\left.-\frac{\Phi(s)}{K}\right)},\right.
\end{aligned}
$$

where $A$ and $B$ are not both 0 .

Then, we choose $s:=(A x+B y) / a(t)$ and the ordinary differential equation:

$$
\ddot{\Phi}(s)-\epsilon^{*} e^{-\frac{\Phi(s)}{K}}=0, \Phi(0)=\alpha, \dot{\Phi}(0)=\beta,
$$

with $\frac{2 \pi e^{C}}{A^{2}+B^{2}}=\epsilon^{*}, \alpha$ and $\beta$ are constants in Lemmas 4 and 5 . Therefore, our solutions (38) satisfy the Poisson equation (4) 3 .

The proof is completed.

Now, we are ready to check that the solutions fit into the Euler-Poisson equations (4). 
Proof of Theorem 1, By Lemma 3 and Lemma 6, the solutions (9) satisfy (4) 1 and (4) 3 . For the $x$-component of the isothermal momentum equations (44) 2 in $R^{2}$, we have

$$
\begin{aligned}
& \rho\left(\frac{\partial u_{1}}{\partial t}+u_{1} \frac{\partial u_{1}}{\partial x}+u_{2} \frac{\partial u_{1}}{\partial y}\right)+\frac{\partial}{\partial x} K \rho+\rho \frac{\partial \Phi}{\partial x} \\
& =\rho\left[\frac{\partial}{\partial t}\left(\frac{\dot{a}(t)}{a(t)} x\right)+\frac{\dot{a}(t)}{a(t)} x \frac{\partial}{\partial x}\left(\frac{\dot{a}(t)}{a(t)} x\right)+\frac{\dot{a}(t)}{a(t)} y \frac{\partial}{\partial y}\left(\frac{\dot{a}(t)}{a(t)} x\right)\right] \\
& +K \frac{\partial}{\partial x} \frac{e^{-\frac{\Phi\left(\frac{A x+B y}{a(t)}\right)}{K}+C}}{a(t)^{2}}+\rho \dot{\Phi}\left(\frac{A x+B y}{a(t)}\right) \frac{A}{a(t)} \\
& =\rho\left[\frac{\ddot{a}(t)}{a(t)} x\right]-\rho \dot{\Phi}\left(\frac{A x+B y}{a(t)}\right) \frac{A}{a(t)}+\rho \dot{\Phi}\left(\frac{A x+B y}{a(t)}\right) \frac{A}{a(t)} \\
& =0
\end{aligned}
$$

by taking $\ddot{a}(t)=0$ that is

$$
a(t)=a_{1}+a_{2} t
$$

For the $y$-component of the isothermal momentum equations (4) 2 , the proof is similar. As the readers may check it, we omit the detail here.

We have shown that the solutions (9) satisfy the Euler-Poisson equations. In particular, $a_{1}>0$ and $a_{2}<0$, the solutions (9) blow up in the finite time $T=-a_{2} / a_{1}$.

The proof is completed.

Remark 7 For the case of $A=0$ and $B=0$, the corresponding solutions (9) may be deduced to the special solutions in [11].

Remark 8 Our solutions (9) also work for the isothermal Navier-Stokes-Poisson equations in $R^{2}$ :

$$
\left\{\begin{aligned}
\rho_{t}+\nabla \cdot(\rho \vec{u}) & =0, \\
(\rho \vec{u})_{t}+\nabla \cdot(\rho \vec{u} \otimes \vec{u})+\nabla K \rho & =-\rho \nabla \Phi+\mu \Delta \vec{u}, \\
\Delta \Phi(t, x) & =2 \pi \rho,
\end{aligned}\right.
$$

where $\mu>0$ is a positive constant.

Additionally, the blowup rate about the solutions is immediately followed:

Corollary 9 The blowup rate of the solutions (9) is,

$$
\lim _{t \rightarrow T} \rho(t, 0,0)(T-t)^{2} \geq O(1)
$$

In conclusion, due to the novel solutions obtained by the separation method, the author conjectures there exists other analytical solution in non-radial symmetry. Further works will be continued for seeking more particular solutions to understand the nature of the Euler-Poisson equations (4). 


\section{References}

[1] M. Bezard, Existence locale de solutions pour les equations d'Euler-Poisson. (French) [Local Existence of Solutions for Euler-Poisson Equations] Japan J. Indust. Appl. Math. 10 (1993), no. $3,431-450$.

[2] J. Binney, S. Tremaine, Galactic Dynamics, Princeton Univ. Press, 1994.

[3] S. Chandrasekhar, An Introduction to the Study of Stellar Structure, Univ. of Chicago Press, 1939.

[4] Y.B. Deng, J.L. Xiang and T. Yang, Blowup Phenomena of Solutions to Euler-Poisson Equations, J. Math. Anal. Appl. 286 (1)(2003), 295-306.

[5] P. Gamblin, Solution reguliere a temps petit pour l'equation d'Euler-Poisson. (French) [Smalltime Regular Solution for the Euler-Poisson Equation] Comm. Partial Differential Equations 18 (1993), no. 5-6, 731-745.

[6] P.Goldreich and S. Weber, Homologously Collapsing Stellar Cores, Astrophys, J. 238, 991 $(1980)$.

[7] J. John and T. Keith, Gas Dynamics, 3rd Edition, Pearson Prentice-Hall, 2006

[8] R. Kippenhahn, A,Weigert, Stellar Sturture and Evolution, Springer-Verlag, 1990.

[9] T. Makino, On a Local Existence Theorem for the Evolution Equation of Gaseous Stars, Patterns and waves, 459-479, Stud. Math. Appl., 18, North-Holland, Amsterdam, 1986.

[10] T. Makino, Blowing up Solutions of the Euler-Poission Equation for the Evolution of the Gaseous Stars, Transport Theory and Statistical Physics 21 (1992), 615-624.

[11] M.W. Yuen, Blowup Solutions for a Class of Fluid Dynamical Equations in $R^{N}$, J. Math. Anal. Appl. 329 (2)(2007), 1064-1079.

[12] M.W. Yuen, Analytical Blowup Solutions to the 2-dimensional Isothermal Euler-Poisson Equations of Gaseous Stars, J. Math. Anal. Appl. 341 (1)(2008), 445-456.

[13] M.W. Yuen, Analytical Blowup Solutions to the Pressureless Navier-Stokes-Poisson Equations with Density-dependent Viscosity in $R^{N}$, Forthcoming Article in Nonlinearity, arXiv:0811.4383v6. 